\title{
Wende, Wolfgang; Tucker, Graham M.; Quétier, Fabien; Rayment, Matt; Darbi, Marianne (Hrsg.) (2018): Biodiversity Offsets. European Perspectives on No Net Loss of Biodiversity and Ecosystem Services
}

\author{
Cham (Schweiz): Springer Nature. 252 S.
}

\section{Adrian Hoppenstedt ${ }^{1}$}

Eingegangen: 11. Juli 2018 / Angenommen: 8. August 2018 / Online publiziert: 15. August 2018

๑) Springer-Verlag GmbH Deutschland, ein Teil von Springer Nature 2018

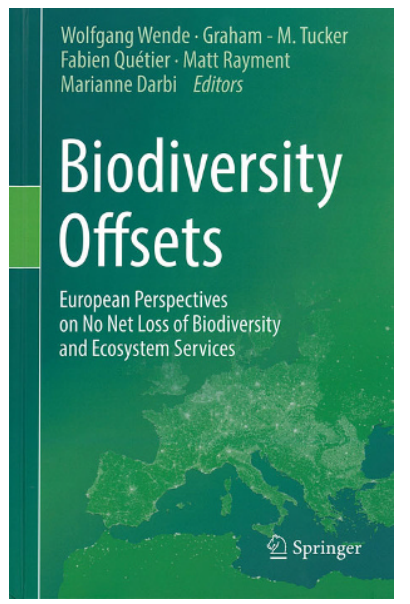

Die fachliche und politische Diskussion um das Erreichen der Ziele der Biodiversitätsstrategie der Europäischen Union (EU) nimmt immer breiteren Raum ein. Zweifelsohne wird es der EU nicht gelingen, die in ihrem ,Action Plan (2017-2019)“ geplanten Ziele zum Stopp des Biodiversitätsverlustes bis $2020 \mathrm{zu}$ erreichen. Daher sind grundlegende wichtige Fragen zu stellen: Welche gesetzlichen Regelungen sind notwendig, welche Instrumente bieten sich an, um den fortschreitenden Verlust von Ökosystemen und Ökosystemleistungen aufzuhalten? Verantwortlich für die Belastungen sind Eingriffe in den Naturhaushalt (vor allem außerhalb von Schutzgebieten) durch verschiedenste Infra-

Prof. Adrian Hoppenstedt

hoppenstedt@hhp-raumentwicklung.de

1 HHP Hage + Hoppenstedt Partner Raum- und Umweltentwicklung, Fridastraße 24, 30161 Hannover, Deutschland strukturen, aber auch die landwirtschaftliche, forstliche und fischereiwirtschaftliche Nutzung.

Das vorliegende Buch versucht - vermutlich erstmalig -, die Erfahrungen mit Kompensationsstrategien von Eingriffen ausgewählter EU-Mitgliedstaaten (Österreich, Belgien, Tschechien, Frankreich, Deutschland, Litauen, Spanien, Niederlande, Großbritannien) und einzelner außereuropäischer Staaten (USA, Australien, Brasilien, Südafrika) zusammenzufassen. Die Vielfalt verschiedener Ansätze und Konzepte zeigt einerseits das verstärkte Bemühen verschiedener Länder, dem Verlust an Biodiversität und Ökosystemleistungen Einhalt zu gebieten, andererseits wird aber durch das Buch sehr gut deutlich, wie unterschiedlich die Herangehensweisen bei Kompensationsregelungen sind und dass es - trotz unterschiedlicher naturräumlicher Bedingungen - dringend einer einheitlichen, zumindest aber vergleichbaren Kompensationsregelung von Eingriffen auf EU-Ebene schon aus Akzeptanzgründen - bedarf. Besonders deutlich werden die Unterschiede bei der Kompensationshierarchie: Vermeidung - Ausgleich - Ersatz. Während beispielsweise in Deutschland und Österreich noch immer der Ansatz der Naturalkompensation gilt und eine Ersatzzahlung die „ultima ratio“ darstellt, steht in vielen Ländern der finanzielle Ersatz im Vordergrund. Bei den Bewertungsmaßstäben, den Bewertungsmethoden, der Einrichtung eines Ökokontos und der Nachkontrolle zeichnen sich Gemeinsamkeiten, aber ebenfalls Unterschiede ab.

Inhaltlich werden in diesem Buch nach einer einheitlichen Struktur die gesetzlichen Rahmenbedingungen, Regularien, die theoretische Herangehensweise und Methodik für ein Verschlechterungsverbot (,No Net Loss“) sowie die Kompensationskonzeptionen der einzelnen ausgewählten neun EU-Länder bei Eingriffen in den Naturhaushalt beschrieben. Hinzu kommen die langjährigen Erkenntnisse mit der Umsetzung der „Principles of the Business and 
Biodiversity Offsets Programme (BBOP)“ der Forstwirtschaft in den USA von 2002 und Erfahrungen anderer Länder im Umgang mit Eingriffen in die Biodiversität.

Zielsetzung des Bandes ist es, einen Beitrag zu einem europäischen Erfahrungsaustausch über bereits bestehende Offset-Politiken, über rechtliche Grundlagen, Methodik und Praxis zu leisten. Auf dieser Grundlage werden letztendlich in einer Zusammenfassung Anforderungen für eine mögliche europäische Eingriffsregelung formuliert. Diese scheint dringend geboten, um die zunehmenden Akzeptanzprobleme in den Bevölkerungen bei divergierenden Kompensationsergebnissen zu reduzieren, aber auch aus Wettbewerbsgründen. Dabei wird hervorgehoben, dass

- Kompensationsregularien auch außerhalb von Schutzgebieten (insbesondere bei Eingriffen durch die Landwirtschaft) notwendig sind,

- eindeutige, gesetzliche Grundlagen geschaffen werden müssen,

- einheitliche Prinzipien, Kriteriengerüste, Standards und Bewertungsmaßstäbe erarbeitet werden müssen,
- es einer vergleichbaren Vermeidungs-/Kompensationshierarchie (Vermeidung, Ausgleich, Ersatz) bedarf,

- möglichst ein einheitliches Vorgehen für die Einrichtung von Ökokonten erarbeitet wird und

- es für eine effektive Umsetzung von Kompensationsmaßnahmen einer Nachkontrolle bzw. eines Monitorings bedarf.

Wie schwierig der Weg noch ist, zeigt der vorläufig misslungene Ansatz einer einheitlichen bundesdeutschen Kompensationsverordnung. Vielleicht bringt ja das von der EU für 2018 angekündigte Dokument zu Hinweisen für einen Ausgleich von Eingriffen in die Biodiversität den erhofften Durchbruch auf dem Weg zu einer europäischen Eingriffsregelung. Das Buch bidlet eine hervorragende Grundlage für eine weitere Diskussion auf dem Weg zur Vereinheitlichung der vielen Erfahrungen mit der Kompensationsproblematik und damit hoffentlich zur Eindämmung des Biodiversitätsverlustes. 